ISSN 0103-5150

Fisioter. Mov., Curitiba, v. 24, n. 1, p. 115-124, jan./mar. 2011 Licenciado sob uma Licença Creative Commons

\title{
Análise da carga de trabalho de analistas de sistemas e dos distúrbios osteomusculares
}

\author{
Analysis of the workload of systems analysts and \\ musculoskeletal disorders
}

\section{Bruno Maia de Guimarães ${ }^{[a]}$, Laura Bezerra Martins ${ }^{[\mathrm{b}]}$, Leonardo Soares de Azevedo ${ }^{[\mathrm{c}]}$, Maria do Amparo Andrade ${ }^{[d]}$}

[a] Especialista, Mestre em Design pela Universidade Federal de Pernambuco (UFPE), Recife, PE - Brasil, email: bmguimaraes@hotmail.com

[b] Doutora, professora da Pós-Graduação em Design da Universidade Federal de Pernambuco(UFPE), Recife, PE - Brasil, email: laurabm@folha.rec.br

[c] Especialista, coordenador da Especialização em Fisioterapia do Trabalho da Faculdade Redentor, Recife, PE - Brasil, email: leoaz@uol.com.br

[d] Doutora, professora do curso de Fisioterapia da Universidade Federal de Pernambuco (UFPE), Recife, PE - Brasil, email: mamparoandrade@yahoo.com

\section{Resumo}

Objetivos: 0 objetivo desta pesquisa foi realizar avaliação das condições ergonômicas de analistas de sistemas, com a finalidade de conhecer os fatores geradores de sobrecargas físicas e cognitivas, suas repercussões sobre os trabalhadores e propor melhorias para a diminuição dessas sobrecargas. Metodologia: Foram utilizados o método SHTM (sistema homem-tarefa-máquina) de avaliação ergonômica; o diagrama de Corlett, para a avaliação do desconforto corporal; o questionário de avaliação do mobiliário; e o NASATLX, para a avaliação de carga mental de trabalho. Resultados: Foram encontradas as prevalências de dor na coluna lombar (71\%) e na coluna cervical (64\%) dos entrevistados. Após a avaliação da carga mental de trabalho, foi observado que a demanda mental foi a mais exigida entre os analistas de sistemas e a média da carga global foi de 13,23. Conclusão: Pode-se concluir que a alta prevalência de dor musculoesquelética pode ser causada pela presença de mobiliário inadequado, pela adoção de posturas incorretas e pela alta exigência mental das atividades desenvolvidas.

Palavras-chave: Carga de trabalho. Analistas de sistemas. Distúrbios osteomusculares. NASA-TLX. 
Abstract

Objective: The purpose of this research was to conduct assessment of ergonomic conditions of systems analysts in order to understand the factors causing physical and cognitive overload, its implications for workers and propose improvements to reduce these physical and cognitive overload. Methodology: The SHTM (humanmachine-task) method was used for ergonomic evaluation, the Corlett diagram for evaluation of bodily discomfort, questionnaire evaluation of the furniture and the NASA-TLX to assess mental workload. Results: It was found a prevalence of pain in the lumbar spine of $71 \%$ and $64 \%$ of respondents in the cervical spine. After evaluation of mental workload, it was observed that the mental demand was most required among systems analysts and the average loading rate was of 13.23. Conclusion: It can be concluded that the high prevalence of musculoskeletal pain may be caused by the presence of inappropriate furniture, adoption of incorrect postures and high mental demand of the developed activities performed by systems analysts.

Keywords: Workload. Systems analysts. Musculoskeletal disorders. NASA-TLX.

\section{Introdução}

O trabalho em larga escala com a utilização do computador, no Brasil, teve início nos últimos 30 anos, alterando definitivamente as relações do homem com o seu trabalho. Atualmente, muitos trabalhadores utilizam a informática em grande parte da sua jornada de trabalho, como é o caso dos analistas de sistemas, que são o objeto desta pesquisa.

Dessa forma, observa-se que as exigências do trabalho informatizado são, principalmente, de atividades que envolvam os processos e as operações cognitivas, tais como monitoração, interpretação, tratamento de informações, resolução de problemas e memória $(1,2)$.

Algumas das várias causas do estresse são a alta exigência mental da tarefa, as grandes responsabilidades assumidas, os conflitos com a chefia, a insatisfação com o trabalho e os fatores organizacionais (3). Nessa perspectiva, em pesquisas de Gredilla e Gonzáles (4), Kawakami et al. (5) e Rocha e Ribeiro (6), os sintomas de estresse foram um fator importante encontrado em analistas de sistemas.

Assim, verifica-se a importância da avaliação da carga mental de trabalho desses profissionais. Tal avaliação é voltada para a medida da carga colocada sobre as habilidades cognitivas do operador (7). De uma forma geral, as técnicas subjetivas são as menos invasivas e possuem alta sensibilidade, tornando-se as mais importantes, e dentre elas destacamse as ferramentas NASA-TLX e SWAT. A literatura vem apresentando pesquisas cujo enfoque é a mensuração de cargas mentais de trabalho, destacando sua importância nas taxas de produtividade, nos acidentes, no absenteísmo e na qualidade de vida no trabalho $(8,9)$.

Além dos problemas relacionados ao estresse, os trabalhadores que utilizam o computador durante grande parte da jornada de trabalho estão sujeitos aos distúrbios musculoesqueléticos relacionados ao trabalho (10-17).

No Brasil, os primeiros casos de LER/DORT surgiram com os digitadores na década de 80 e, a partir daí, a ocorrência desse grupo de doenças vem aumentado. No início da década de 90, as LER/DORT, juntamente com a surdez, tornaram-se as doenças do trabalho mais notificadas pelo INSS e as que mais demandaram serviços de saúde ao trabalhador (18). Segundo dados da Previdência Social, observa-se que, em 2002, foram registrados aproximadamente 22 mil casos de LER/DORT; em 2003, 23 mil; e em 2004 em torno de 27 mil casos. Ainda segundo o Ministério da Previdência Social, os gastos em 2005 com o pagamento de benefícios decorrentes de acidentes de trabalho atingiram a quantia de $\mathrm{R} \$ 9,8$ bilhões. Ainda assim, verifica-se que é consensual a opinião dos que atuam na área de que há uma subnotificação de cerca de $80 \%$ dos acidentes do trabalho, sendo boa parte destes contabilizados entre os segurados da Previdência Social que recebem benefícios não acidentários (19).

Graças ao alto custo no tratamento das LER/DORT e ao grande número de subnotificações, o Decreto n. 6.042 da Previdência Social entrou em vigor em abril de 2007 e regulamentou as mudanças na caracterização das doenças e acidentes relacionados ao trabalho, pelo novo sistema de nexo técnico epidemiológico (NTEP). Assim, as doenças ocupacionais 
passaram a ser caracterizadas tecnicamente pela perícia médica do INSS mediante a identificação do nexo causal entre o trabalho e a doença, quando se verifica a relação entre a atividade da empresa identificada pela Classificação Nacional de Atividade Empresarial (CNAE), e a doença ou sequela que motivou a incapacidade - identificada pelo Código Internacional de Doenças (CID).

A partir desse momento, a tendência é um grande aumento do número de notificações de casos de LER/DORT entre os trabalhadores, em razão da grande quantidade de casos que anteriormente eram subnotificados e também pela notificação de casos nos quais existe a relação do CNAE da empresa com o CID do distúrbio musculoesquelético do trabalhador, mesmo ele não exercendo atividade que poderia causar a lesão. Em corroboração, dados do INSS de 2007 mostram que houve aumento de 148\%, em relação ao ano anterior, na concessão de auxíliosdoença de natureza acidentária, de que fazem parte as LER/DORT (20).

Os fatores causadores ou agravantes das LER/ DORT têm sido bem discutidos e podem ser agrupados da seguinte forma: fatores físicos e biomecânicos, como posturas inadequadas, repetitividade de movimentos, velocidade exercida durante a tarefa, iluminação, ruídos e outros; fatores organizacionais, como pausas, ritmos, sazonalidade da produção, estruturas de horários, métodos impróprios de trabalho, forma da produção e outros; fatores individuais, como gravidez, doenças crônicas como artrite e diabetes, sexo, hereditariedade, prática de esportes, entre outros; e, por fim, fatores psicossociais, tais como satisfação no trabalho, relacionamento com os colegas, ansiedade e expectativa individual (21).

O trabalho informatizado impõe ao corpo posturas paradoxais: enquanto alguns segmentos corporais permanecem estáticos por longos períodos de tempo, como a coluna vertebral, outros segmentos, como os membros superiores, precisam realizar movimentos altamente repetitivos, impedindo igualmente a recuperação dos tecidos e das estruturas fisiológicas envolvidas nessa manutenção postural (22). Quando esse trabalho é realizado diariamente, ao longo de várias horas da jornada laboral, predispõe ao trabalhador o aparecimento de lesões. Dessa forma, verifica-se a necessidade de mudanças posturais no decorrer da jornada de trabalho, como, por exemplo, a adoção de posturas como: ficar em pé, sentado, flexionado para frente e reclinado para trás.
0 mobiliário de trabalho é um fator importante para promover o conforto, a segurança e a eficiência dos trabalhadores, no caso dos analistas de sistemas que realizam praticamente todas as suas atividades de trabalho utilizando o computador. Torna-se, assim, ainda mais importante um mobiliário adequado às características dos usuários, das tarefas e dos fatores organizacionais. Assim, os critérios para a definição dos parâmetros do mobiliário dos postos de trabalho são selecionados a partir de observação do modo de execução da atividade, identificação das características físicas do ambiente e exigências relacionadas à organização do trabalho (23).

É importante observar que a aquisição de mobiliário ergonomicamente correto não é garantia suficiente para a solução dos problemas físico-posturais do trabalho $(24,25)$, sendo de muita importância os aspectos relacionados à organização deste. Dessa forma, quando o trabalho é conflituoso e desorganizado, propicia um acúmulo de tensão que pode ter repercussões sobre o corpo (26).

0 fato de a automação e a informatização no mundo do trabalho trazerem consigo tarefas cujas exigências são de natureza predominantemente cognitivas solicita da ergonomia o desenvolvimento de novas abordagens teórico-metodológicas para apreender o funcionamento do homem de forma situada $(2,27,28)$.

Assim, diante desse importante problema de saúde ocupacional, verifica-se a importância de avaliar as condições de trabalho dessa classe de trabalhadores, a fim de conhecer os fatores geradores de sobrecargas físicas e cognitivas e suas repercussões sobre os trabalhadores e propor melhorias para a diminuição dessas sobrecargas.

\section{Métodos}

O método de avaliação ergonômica utilizado foi o SHTM, descrito por Moraes e Mont'Alvão (29), o qual compreende cinco fases: apreciação ergonômica; diagnose ergonômica; projetação ergonômica; avaliação e validação ergonômica; e detalhamento ergonômico e otimização. A proposta de intervenção ergonomizadora pode compreender apenas a $1^{\underline{a}}$ etapa ou a continuação, em sequência, de todas as etapas, o que dependerá das necessidades e objetivos do projeto em questão. A presente pesquisa englobou as fases de apreciação e diagnose ergonômica. 
A apreciação ergonômica é uma fase exploratória que compreende o mapeamento dos problemas ergonômicos da empresa. Consiste na sistematização do sistema homem-tarefa-máquina e na delimitação dos problemas ergonômicos. São realizadas observações assistemáticas no local de trabalho, entrevistas com os funcionários e registros fotográficos da tarefa. É nesta fase que se identifica a provável causa do problema a ser enfocado na diagnose. Todos esses procedimentos foram realizados em momentos convenientes para a empresa e para os trabalhadores sem provocar qualquer alteração ou perturbação na rotina de trabalho destes.

A diagnose ergonômica permite aprofundar os problemas priorizados na apreciação e termina com as recomendações ergonômicas aos postos de trabalho avaliados. Os instrumentos de diagnose utilizados foram: questionário para a avaliação do mobiliário, descrito por Couto (30); diagrama de desconforto corporal, sugerido por Corlett e Bishop (31); e avaliação de carga mental de trabalho por meio do NASA-TLX (Task Load Index), desenvolvido por Hart e Staveland, (32).

O NASA-TLX, como método multidimensional, demonstrou grande eficiência e praticidade quando comparado a outros métodos unidimensionais precedentes a ele (33). O NASA-TLX é um procedimento multidimensional que fornece uma avaliação quantitativa global da carga mental de trabalho, baseada na média ponderada da avaliação de seis dimensões dessa carga: exigência mental; exigência física; exigência temporal; nível de realização; nível de esforço; e nível de frustração. Esses conceitos são apresentados ao sujeito antes da aplicação do instrumento.

Destaca-se que o NASA-TLX apresenta a particularidade de ponderar as dimensões da carga mental de trabalho, de acordo com a importância subjetiva (peso) atribuída pelo sujeito, multiplicada pela taxa aferida para cada dimensão da carga, visto que as dimensões da carga de trabalho variam de acordo com as tarefas/atividades e a forma como o sujeito as percebe. 0 grau com que cada uma das dimensões contribui para essa carga mental pode ser determinado pelo valor das taxas. Cada taxa é determinada a partir de um valor numérico aferido em uma folha que contém as seis dimensões em escalas graduadas sem valores numéricos. 0 sujeito assinala como ele percebe em que magnitude determinada dimensão contribuiu para a formação da carga mental de trabalho na tarefa, e o examinador posteriormente identifica o valor numérico vinculado ao nível assinalado pelo sujeito. Cada escala apresenta uma linha de $15 \mathrm{~cm}$, dividida em 20 partes iguais ancoradas em descrições bipolares (baixa e alta).

0 peso com que cada dimensão contribui para a carga mental de trabalho é aferido por meio de um conjunto de confrontos das dimensões dessa carga, em que são apresentados 15 pares das dimensões combinadas (todas as combinações possíveis). 0 sujeito deve escolher a dimensão que mais contribui para a carga mental de trabalho que ele percebe durante a execução da tarefa. Desse modo, cada dimensão pode ser selecionada desde nenhuma vez até cinco vezes. As taxas e os pesos de cada dimensão são obtidos após o sujeito ter efetuado a tarefa ou parte dela. As dimensões que apresentam maior peso na origem da carga mental de trabalho apresentarão maior peso na pontuação da carga de trabalho global, dando, dessa forma, um implemento em sensibilidade para a escala. Ao final do procedimento, é calculada a taxa global ponderada da carga mental de trabalho do sujeito. Essa taxa global ponderada é obtida por meio do somatório de todos os pesos multiplicados pelas taxas de todas as dimensões e esse valor é dividido por 15 , oferecendo o valor final.

A amostra do estudo foi composta por 45 analistas de sistemas de um escritório de informática na cidade de Recife. Os dados foram coletados, tabulados, processados e analisados no software Microsoft ${ }^{\circledR}$ Excel 2003. O presente estudo foi aprovado pelo Comitê de Ética em Pesquisa do Centro de Ciências da Saúde da Universidade Federal de Pernambuco (CEP/CCS/UFPE), registro n. 030/07, e encontra-se de acordo com as normas vigentes.

\section{Resultados}

\section{Apreciação ergonômica}

O estudo foi realizado numa empresa pública federal de desenvolvimento de software, sediada na cidade de Recife, PE, composta por 128 analistas de sistemas. A amostra estudada foi escolhida aleatoriamente e formada por 45 analistas de sistemas com idade média de 31 anos, distribuídos em 30 homens e 15 mulheres. A jornada diária de trabalho é de 8 horas de segunda a sexta-feira, inicia-se às 8 
horas da manhã e termina às 17 horas com intervalo para o almoço de 60 minutos. A empresa possui pausas para realização de ginástica laboral nos turnos da manhã e da tarde, de segunda a sexta-feira, para todos os funcionários, com duração média de 20 minutos.

Os analistas de sistemas da empresa pesquisada realizam as atividades de desenvolvimento, avaliação, teste e implementação de software por meio de pequenos grupos com cerca de quatro trabalhadores cada. Para realizar as atividades de trabalho, é necessário o uso do computador na maior parte do tempo. As reuniões ocorrem com frequência entre as equipes, para a definição de metas e procedimentos e a avaliação do andamento do trabalho. Assim, verifica-se que há uma frequente exigência mental no desenvolvimento das atividades de trabalho.

\section{Problemas identificados}

Os problemas foram identificados por meio de observação direta dos pesquisadores, durante as visitas ao local, de fotografias, de filmagens e de entrevistas informais com os trabalhadores e supervisores.

Foram encontrados problemas posturais durante a realização das atividades de trabalho, em que os trabalhadores pesquisados adotavam posturas sentadas inadequadas como, por exemplo, sentado inclinado para frente (Figura 1) e sentado sem apoiar as costas no apoio da cadeira. Outras posturas incorretas para a realização de trabalhos em terminal de vídeo foram observadas, como a flexão cervical acentuada durante a visualização do monitor e braços não apoiados na bancada de trabalho durante o uso do teclado e do mouse.

Em relação ao mobiliário de trabalho, verificouse que muitos monitores estavam baixos (Figura 2), ou seja, posicionados abaixo do recomendado por diversos autores (altura da linha dos olhos do usuário) (34-36). Foi encontrada em alguns postos de trabalho, a ausência de espaço para apoio dos braços durante o manuseio do teclado e do mouse. As bancadas de trabalho não apresentavam regulagens de altura, não se adequando aos trabalhadores com elevada e baixa estatura. Não foram encontrados problemas relacionados à cadeira, pois as que são usadas pelos analistas de sistemas pesquisados são de boa qualidade e apresentam as regulagens necessárias para a adoção de uma boa postura de trabalho.

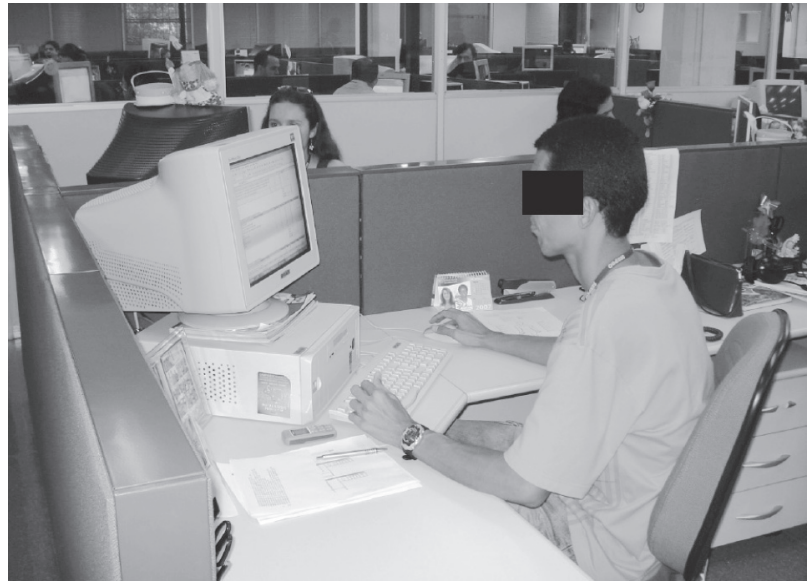

Figura 1 - Postura inadequada (sentado inclinado para frente, sem apoiar as costas no apoio da cadeira)

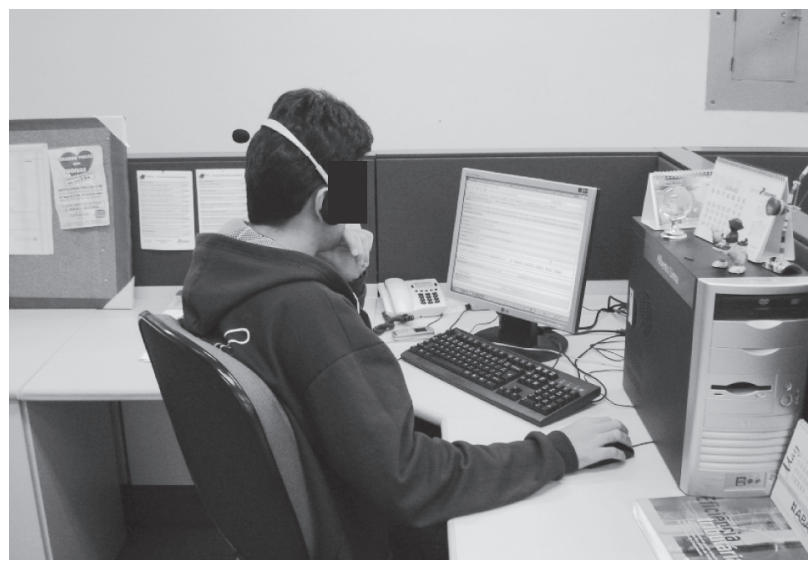

Figura 2 - Mobiliário de trabalho (monitor baixo, ou seja, posicionado abaixo da linha dos olhos do usuário)

Verificou-se a ausência de apoio para os pés dos funcionários de baixa estatura, uma vez que a bancada de trabalho não apresenta regulagem de altura, o trabalhador necessita regular a cadeira na altura máxima para o uso do teclado e do mouse e, assim, não alcança o chão com os pés. Essa situação pode provocar compressão na região posterior da coxa, dificultando a circulação sanguínea nos membros inferiores $(36,37)$.

Em relação aos fatores organizacionais do trabalho, vários trabalhadores em entrevistas informais afirmaram que os prazos para a execução do trabalho eram curtos e que era frequente a realização de horas extras à noite e nos fins de semana. Apesar de esses fatores não terem sido mensurados nesta pesquisa, é importante verificar a sua importância, visto que a alta frequência de estresse nos analistas 
de sistemas é causada principalmente por prazos curtos para desenvolvimento das tarefas e sobrecarga de trabalho, principalmente sobrecarga mental $(6,38)$.

Após o levantamento inicial dos problemas encontrados pelos pesquisadores, iniciou-se a diagnose ergonômica, na qual, por meio dos questionários, procurou-se aprofundar e confirmar os problemas encontrados na apreciação.

\section{Diagnose ergonômica}

\section{Análise dos questionários}

A partir da análise dos questionários referentes às dores sentidas atualmente em regiões do corpo pelos entrevistados, foi possível obter o Gráfico 1, que aparece na sequência. A região do corpo onde a maioria dos entrevistados $(n=45)$ afirmou sentir dor foi a região da coluna lombar (71\%), seguida pela coluna cervical (64\%). Esses resultados são semelhantes aos encontrados por Rocha e Ribeiro (6) em analistas de sistemas que tinham maior prevalência de dor nas regiões da coluna lombar (57\%) e cervical/ombros (55\%) nos homens, e, nas mulheres, de $74 \%$ nas regiões cervical/ombros e 72\% na coluna lombar. Campos (39) também encontrou resultados semelhantes com trabalhadores do setor de informática, em que as regiões com maior frequência de dor foram: coluna lombar $(43,67 \%)$, ombro $(37,34 \%)$ e coluna cervical $(34,81 \%)$.

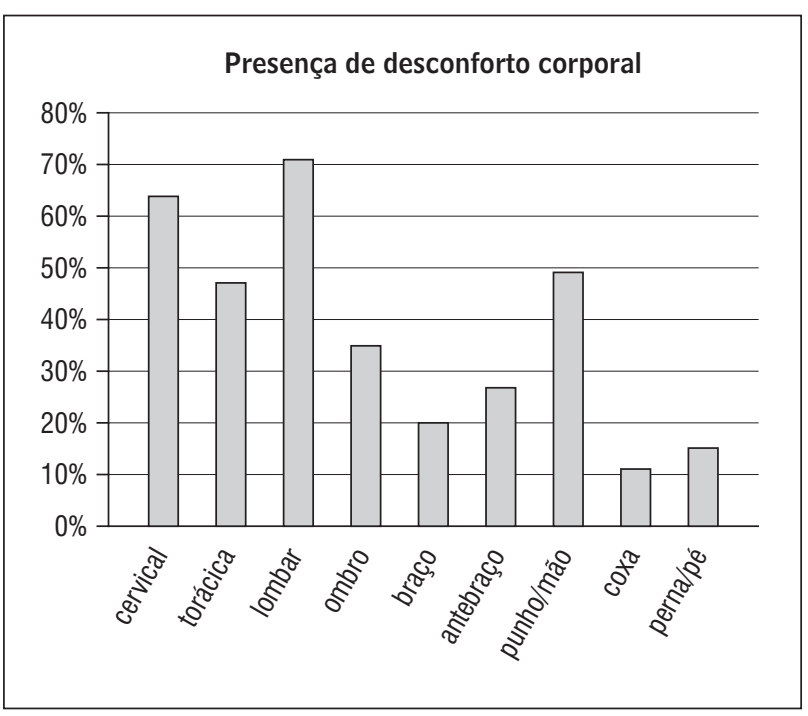

Gráfico 1 - Prevalência de dor nos entrevistados
Na avaliação do mobiliário de trabalho quanto ao conforto e à posição dos itens analisados, obteve-se o Gráfico 2. Em relação à cadeira, 35\% dos entrevistados afirmaram que ela é boa, $40 \%$ relataram que é regular e $25 \%$ classificaram como ruim. Aproximadamente $55 \%$ dos participantes apontaram como boa a bancada de trabalho, 30\% como regular e $15 \%$ como ruim. A posição do teclado foi avaliada como boa em $40 \%$ dos casos, $35 \%$ como regular e $25 \%$ como ruim. Com relação ao posicionamento do mouse, 35\% dos entrevistados afirmaram que é bom, $40 \%$ que é regular e $25 \%$ que é ruim. A posição do monitor foi classificada como boa por $29 \%$ dos entrevistados, $38 \%$ como regular e $33 \%$ como ruim.

Assim, percebe-se que o posicionamento do monitor foi o item que mais incomodou os usuários. A partir desses dados e com a ocorrência de vários monitores posicionados abaixo da linha dos olhos dos trabalhadores, o que foi observado na fase de apreciação, existe a possibilidade de se relacionar isso com a importante prevalência de dor encontrada na coluna cervical. Esses dados corroboram com Chaffin e Anderson (36), os quais concluíram que a tela do monitor, estando abaixo da linha dos olhos, aumenta a flexão da coluna cervical, causando aumento da exigência dos músculos extensores do pescoço para suportar o peso da cabeça.

A cadeira não foi um dos itens mais citados pelos entrevistados como um fator de desconforto, porém, por meio do questionário de dor, foi encontrada prevalência de dor na região lombar em 71\% dos entrevistados. Isso pode estar relacionado com a postura ao sentar e com a permanência prolongada na posição "sentado". Os distúrbios lombares, em decorrência da postura sentada, podem ser justificados pelo fato de a compressão dos discos intervertebrais ser $40 \%$ maior na posição "sentado" que na posição "em pé", porém, tais problemas não são apenas decorrentes das cargas que atuam sobre a coluna vertebral, mas principalmente da manutenção da postura estática $(30,40)$.

A adoção de postura adequada ao sentar é muito importante para reduzir a sobrecarga na coluna lombar e, para isso, é necessário manter um bom ângulo entre o tronco e as coxas, que deve ser necessariamente maior que $90^{\circ}$ e menor que $110^{\circ}(23$, 30, 40-42).

A avaliação da carga mental de trabalho por meio do NASA-TLX pode ser evidenciada nos Gráficos 3 e 4. Verifica-se no Gráfico 3 que a demanda que apre- 
senta a maior área no gráfico - ou seja, a que mais contribui para a formação da carga mental global foi a demanda mental, com 67,95, e a menor contribuição ficou por conta da física, com 0,66. A carga mental global média dos trabalhadores foi de 13,23, como está evidenciado no Gráfico 4.

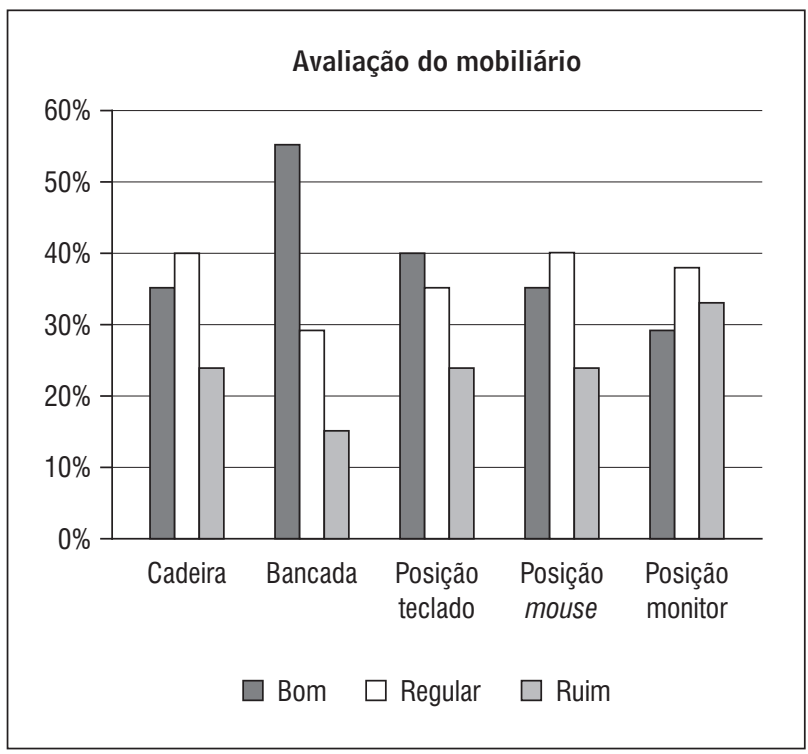

Gráfico 2 - Gráfico resultante da avaliação do mobiliário pelos trabalhadores

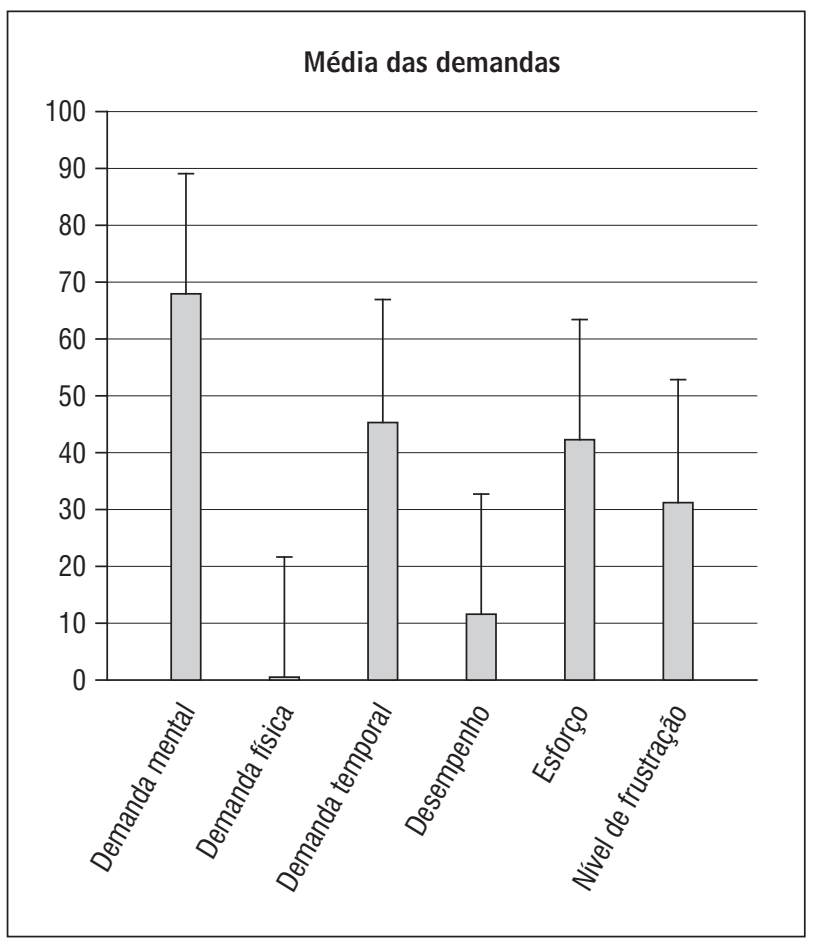

Gráfico 3 - Gráfico resultante da média das demandas obtida pelo NASA-TLX

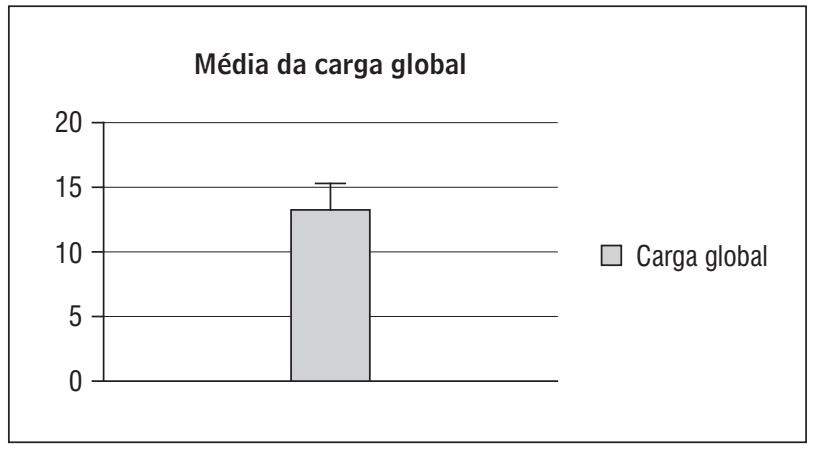

Gráfico 4 - Gráfico resultante da média da carga mental global obtida pelo NASA TLX

Assim, a partir dos resultados obtidos após a aplicação do NASA-TLX, percebe-se que a demanda mental é o fator mais exigido no trabalho dos analistas de sistemas, sendo o que mais contribuiu para a formação da carga mental global. Esses dados estão de acordo com os resultados encontrados por Rocha e Ribeiro (6), que em pesquisa com analistas de sistemas da região metropolitana de São Paulo encontraram a frequência de $59,1 \%$ e $76,4 \%$ para a fadiga mental em homens e mulheres, respectivamente.

Essa importante carga mental encontrada pode ser causada pela característica do trabalho, por ser uma tarefa de grande exigência de concentração, raciocínio, tomada de decisão e memória. Os principais fatores de incômodo e fadiga relatados por analistas de sistemas foram: prazos curtos, uso constante da mente e alto grau de responsabilidade (6).

É importante salientar que há uma constatação frequente: a de que os trabalhadores que desempenham tarefas com carga mental predominante queixam-se de perturbações físicas, tais como dores nas costas e no pescoço, corroborando os resultados encontrados nesta pesquisa (29).

\section{Recomendações}

Os benefícios da intervenção ergonômica em postos de trabalho informatizados se dão, principalmente, pela diminuição dos desconfortos musculoesqueléticos dos trabalhadores e por tornar o ambiente mais confortável e adequado para o trabalho $(14,16,34,43-47)$. Para isso, é necessário disponibilizar suporte de altura regulável para monitor no qual a sua borda superior fique na mesma altura dos olhos do usuário. Assim, evita-se a manutenção 
da flexão cervical durante a visualização do terminal de vídeo, diminuindo a exigência dos músculos extensores do pescoço para suportar o peso da cabeça. Verifica-se a importância de trocar as bancadas de trabalho, tendo em vista que as atuais não apresentam regulagens de altura, possibilitando, assim, que os usuários possam regular a altura da mesa de trabalho a suas dimensões corporais, levando ao maior conforto durante a execução das atividades. É necessário fornecer apoio apropriado para os pés, com regulagens de altura e inclinação, aos funcionários de baixa estatura, para dessa forma evitar que haja compressão da cadeira sobre a região posterior da coxa, dificultando a circulação sanguínea nos membros inferiores. É importante também realizar um estudo de avaliação dos prazos para a execução das tarefas para se verificar se estão de acordo com as capacidades dos trabalhadores para, dessa forma, diminuir a sobrecarga mental do trabalho, evitando o surgimento do estresse.

\section{Considerações finais}

Muitos dos problemas relatados até aqui, referentes aos constrangimentos posturais e à própria organização do trabalho, podem ser decorrentes da desinformação dos funcionários e da própria administração. Esses problemas poderiam ser minimizados com um trabalho de orientação sobre organização do trabalho e um treinamento para o uso correto do mobiliário.

Contudo, é importante ressaltar que somente o fornecimento de mobiliário adequado e a adoção de posturas corretas não são suficientes para eliminar a presença dos distúrbios músculo-esqueléticos relacionados ao trabalho, no caso de os funcionários estarem sujeitos a altas exigências cognitivas. As sobrecargas mentais que foram encontradas nos sujeitos desta pesquisa, como, por exemplo, a necessidade de concentração, de raciocínio, de tomada de decisão e memória, para o desenvolvimento das atividades de trabalho, em associação à pressão temporal podem levar ao estresse.

Atualmente, o estresse é um fator amplamente discutido e pesquisado por diversos cientistas ao redor do mundo. Sabe-se que uma das repercussões do estresse no corpo humano é sobre o sistema músculo-esquelético, que, graças à liberação de substâncias químicas pelo organismo na corrente sanguínea, pode provocar o aumento da tensão muscular. Esse aumento ocorre principalmente na região do pescoço e/ou da coluna lombar, podendo levar a alterações na circulação sanguínea da região e dos membros superiores e inferiores, predispondo o surgimento de distúrbios musculoesqueléticos e as LER/DORT.

Assim, pode-se concluir, por meio dos resultados encontrados e da literatura pesquisada, que a alta prevalência de dor musculoesquelética, principalmente na coluna lombar e cervical, entre os analistas de sistemas, pode ser causada pela presença de mobiliário inadequado, pela adoção de posturas incorretas e pela alta exigência mental das atividades desenvolvidas.

\section{Referências}

1. Sperandio JC. L'ergonomie du travail mental. Paris: Masson; 1984.

2. Rasmussen J. Human factors in a dynamic information society: where are we heading? Ergonomics. 2000; 43(7):869-79.

3. Iida I. Ergonomia: projeto e produção. 2a ed. São Paulo: Edgard Blücher; 2005.

4. Gredilla JMC, Gonzalez JM. Vigilancia médica específica en los trabajadores de pantallas de visualización de datos. Salud Trab. 1991;84:9-16.

5. Kawakami N, Roberts CR, Haratani T. Effects of job stressors on physical and mental health in Japanese VDU workers. In: Proceedings the of 5th International Scientific Conference on Work With Display Units; 1997; Tokyo, 1997. p. 127-8.

6. Rocha LE, Debert-Ribeiro M. Trabalho, saúde e gênero: estudo comparativo sobre analistas de sistemas. Rev. Saúde Pública, 2001;35(6):539-47.

7. Alves JLL. Avaliação da carga mental de trabalho na operação de interfaces homem-computador de sistemas de controle de processo [tese]. São Paulo: Escola Politécnica, Universidade de São Paulo; 2004.

8. Sato N, Kamada T, Miyake S, Akatsu, J, Kumashiro M, Kume Y. Subjective mental workload in type A women. Int J Ind Ergon. 1999;24(3):331-6.

9. Miyake S. Multivariate workload evaluation combining physiological and subjective measures. Int J Psychophysiol. 2001;40(3):233-8. 
10. Hunting W, Laubli T, Grandjean E. Postural and visual loads at VDT workplaces. Constrained postures. Ergonomics. 1981;24:917-31.

11. Bergqvist U, Wolgast E, Nilsson B, Voss M. Musculoskeletal disorders among visual display terminal workers: individual, ergonomic, and work organizational factors. Ergonomics, 1995;38:763-76.

12. Maybe I, Fall MC, Sagnon A, Sow ML. Survey of pathology associated with the use of video display terminals. Dakar Med. 1998;43(1):37-40.

13. Turville KL, Psihogios JP, Ulmer TR, Mirka GA. The effects of video display terminal height on the operator: a comparison of the $15^{\circ}$ and $40^{\circ}$ recommendations. Appl Ergon. 1998;29(4):239-46.

14. Psihogios JP, Sommerich CM, Mirka, GA, Moon SD. A field evaluation of monitor placement effects in VDT users. Appl Ergon. 2001;32(4):313-25.

15. Korhonen $\mathrm{T}$, Ketola R, Toivonen R, Luukkonen R, Hakkanen M, Viikari-Juntura E. Work related and individual predictors for incident neck pain among office employees working with video display units. Occup Environ Med. 2003;60(7):475-82.

16. Sillampã J, Huikko S, Nyberg M, Kivi P, Laippala $P$, Uitti J. Effect of work with visual display units on musculo-skeletal disorders in the office environment. Occup Méd. 2003;53(7):443-51.

17. Kristensen BJ, Jensen C. Self reported workplace related ergonomic conditions as prognostic factors for musculoskeletal symptoms: the "BIT" follow up study on office workers. Occup Environ Med. 2005; 62(3):188-94.

18. Núcleo de Referência em Doenças Ocupacionais da Previdência Social - NUSAT. Relatório Anual. Belo Horizonte: NUSAT; 1993.

19. Maeno M. Reestruturação capitalista [acesso em 15 jan. 2010]. Disponível em: http://www.fazer.com. br/layouts/abrat/default2.asp?cod_materia $=2484$

20. Ministério da Previdência e Assistência Social. Divisão de Planejamento e Estudo Estratégicos. Boletim Estatístico de Acidentes de Trabalho-BEAT [acesso em 20 mar. 2007]. Disponível em: www.mpas.gov.br/pg seundarias/paginas_perfis/perfil_Empregador_10_ 04-A5.asp
21. Coury HJCG, Walsh IAP, Pereira ECL, Manfrim GM, Perez L. Indivíduos portadores de LER acometidos há 5 anos ou mais: um estudo da evolução da lesão. Rev Bras Fisioter. 1999;3(2):79-86.

22. Coury HJCG. Perspectivas e requisitos para a atuação preventiva da fisioterapia nas lesões músculoesqueléticas. Fisioter Mov. 1993;5(2):63-8.

23. Kroemer KHE, Grandjean E. Manual de ergonomia: adaptando o trabalho ao homem. 5a ed. Porto Alegre: Bookman; 2005.

24. da Silva RM, Moreira JA. Uma análise da utilização de mobiliário ergonômico - caso: Telpa SA. Anais do Encontro Nacional de Engenharia de Produção, Piracicaba; 1996.

25. Brasil. Ministério do Trabalho. Manual de Aplicação da Norma Regulamentadora n. 17. 2a ed. Brasília: Secretaria de Inspeção do Trabalho; 2002.

26. Dejours C, Abdoucheli E, Jayet C. Psicodinâmica do trabalho: contribuições da escola dejouriana à análise da relação prazer, sofrimento e trabalho. São Paulo: Atlas; 1994

27. Marmaras N, Pavard B. Problem-driven approach to the design of information technology systems supporting complex cognitive tasks. Cognition, Technology \& Work. 1999;1:222-36.

28. Marmaras N, Kontogians T. Cognitive task. In: Salnend G. Handbook of industrial engineering. New York: John Wiley \& Sons; 2001.

29. Moraes A, Mont'alvão C. Ergonomia: conceitos e aplicações. 2a ed. Rio de Janeiro: 2AB; 2002.

30. Couto HA. Ergonomia aplicada ao trabalho. Belo Horizonte: Ergo; 1995.

31. Corlett EN, Bishop RP. A technique for assessing postural discomfort. Ergonomics. 1976;19(2):175-82.

32. Hart SG, Staveland LE. Development of a NASA-TLX (Task Load Index): results of empirical and theoretical research. In: Hancock PA, Meshkati N, editor. Human mental workload. Amsterdam: Elsevier; 1988.

33. Hendy KC, Hamilton KM, Landry LN. Measuring subjective workload: when is one scale better than many? Hum Factors. 1993;35(4):579-601. 
34. Limerick RB, Plooy A, Ankrum DR. The effect of imposed and self selected computer monitor height on posture and gaze angle. Clin Biomech. 1998;13(8): 584-92.

35. Mon-Williams M,LimerickRB,PlooyA,WannJ.Vertical gaze direction and postural adjustment: an extension of the Heuer model. J Exp Psychol Appl. 1999; 5(1):35-53.

36. Chaffin DB, Anderson GB. Occupational biomechanics. 2a ed. New York: Wiley; 2001.

37. Mairiaux PH. Polígrafo curso Ergonomia, UCL - Universidade Católica de Louvain: Cap. V: A postura de trabalho, Cap. VI: Concepção do posto de trabalho, Bélgica; 1992.

38. Merlo ARC. A informática no Brasil: prazer e sofrimento no trabalho. Porto Alegre: Ed. da UFRGS; 1999.

39. Campos, ES. Distúrbios músculo-esqueléticos em trabalhadores da área de informática: um enfoque à dor lombar [dissertação]. Santa Catarina: Programa de Pós-Graduação em Engenharia de Produção, Universidade Federal de Santa Catarina; 2004.

40. Rio RP, Pires L. Ergonomia: fundamentos da prática ergonômica. Belo Horizonte: Health; 1999.

41. Sanders M, Cormick JMC. Human factors in engineering and design. 7a ed. New York: McHill, Inc; 1993.

42. Coury HJCG. Trabalhando sentado: manual para posturas confortáveis. São Carlos: UFSCar; 1994.
43. Kietrys DM, McClure PW, Fitzgerald GK. The relationship between head and neck posture and VDT screen height in keyboard operators. Phys Ther. 1998;78(4):395-404.

44. Dowler E, Kappes B, Fenaughty A, Pemberton G. Effects of neutral posture on muscle tension during computer use. Int J Occup Saf Ergon. 2001;7(1): 61-78.

45. Ketola R, Toivonen R, Häkkänen M, Luukkonen R, Takala EP, Viikari-Juntura E. Expert group in ergonomics. Effects of ergonomic intervention in work with video display units. Scand J Work Environ Health. 2002;28(1):18-24.

46. Marcus M, Gerr F, Monteilh C, Ortiz DJ, Gentry E, Cohen $\mathrm{S}$, et al. A prospective study of computers users: II. Postural risk factors for musculoskeletal symptoms and disorders. Am J Ind Méd. 2002;41(4):236-49.

47. Travers PH, Stanton BA. Office workers and video display terminals: physical, psychological and ergonomic factors. AAOHN J. 2002;50(11):489-93.

Recebido: $14 / 07 / 2010$ Received: 07/14/2010

Aprovado: $15 / 11 / 2010$ Approved: 11/15/2010 